\title{
Was bringen topische Steroide bei chronischer Rhinosinusitis?
}

\section{Zur Behandlung der chronischen Rhinosinusitis gehören meist auch intranasal applizierte Steroide. Während ihre positive Wirkung bei Erkrankungen mit Polyposis nasi in zahlreichen Studien beschrieben ist, sind die Untersuchungsergebnisse für Formen ohne Nasenpolypen weniger eindeutig, wie australische Wissenschaftler nun in einer Übersichtsarbeit zeigten.}

anz überzeugt sind die Forscher noch nicht davon, dass topische Kortikoide auch bei chronischer Rhinosinusitis (CRS) ohne Polypen einen Nutzen bringen. Bei ihrer Suche nach Studien zu diesem Thema fanden sie nur wenige, obwohl sie ihre Einschlusskriterien recht weit fassten. Letztendlich gingen neun Arbeiten in ihre Übersicht ein, wobei der Studienaufbau recht unterschiedlich war und lediglich eine Studie bezüglich der Qualität offiziell anerkannten Standards genügte. Ein Problem war außerdem, dass die Definition der CRS nicht einheitlich war. Acht Arbeiten verglichen die Wirkung topischer Steroide mit der von Placebo, eine beurteilte die Wirkung zweier unterschiedlicher Dosierungen. Eingesetzt wurden Beclomethason, Fluticason, Budesonid, Dexamethason und Tixocortol. Die Medikamente wurden meist nasal, in einem Fall jedoch direkt in einen Sinus maxillaris appliziert. Die Dauer der Beobachtung lag zwischen elf Tagen und einem Jahr, wobei nur zwei Studien bis zur Beendigung der Therapie fortgeführt wurden. Fünf Arbeiten beurteilten einen Gesamtnutzen der Therapie auf eine Kombination von Symptomen und klinischen Parametern. Eine gemeinsame Auswertung der entsprechenden Werte zeigte keinen signifikanten Nutzen der topischen Kortikoide, wobei eine hohe Heterogenität der Daten auffiel. Die Wirkung auf die Gesamtsymptomatik erfassten insgesamt drei Studien, deren Zusammenschau einen Therapieerfolg zeigte. Übereinstimmung zeigten die Studien, was Nebenwirkungen anging: Keine zeigte diesbezüglich einen Unterschied zwischen Verum und Placebo, vermehrte Infektionen durch die Therapie wies ebenso keine der Studien nach.

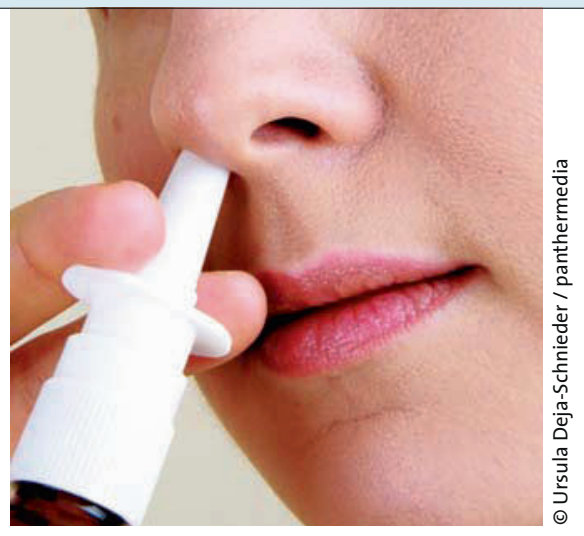

Wie wirksam sind Kortikosteroide bei chronischer Rhinosinusitis?

Fazit: Die vorliegenden Daten seien nicht ausreichend, um von einem Gesamtnutzen der Kortikoide auszugehen, erklären die Autoren. Möglich sei jedoch, dass die Therapie zu einer Symptomverbesserung führe. Da die Negativwirkungen nach bisherigen Untersuchungen sehr gering seien, sollten die lokalen Kortikoide weiterhin ein Teil der Behandlung der CRS ohne Polyposis nasi bleiben. Als Mittel der Wahl empfehlen die Wissenschaftler Budenosid, das als einziges Medikament in einer Arbeit einen klaren Nutzen bewiesen hatte. ib

Kalish LH et al. Topical steroids in chronic rhinosinusitis without polyps: a systematic review and meta-analysis. Otolaryngol Head Neck Surg. 2009; 141(6): 674-83. 